\title{
3 themes related to a critical incident were identified in the decision to place a relative with Alzheimer's disease in an assisted living facility
}

\author{
Liken MA. Critical incidents precipitating institutionalization of a relative with Alzheimer's. West J Nurs Res 2001 \\ Mar;23:163-78.
QUESTION: What precipitates the decision of caregivers of people with Alzheimer's disease (AD) to place their relatives in an assisted living facility (ALF)?

Design

Secondary analysis of data collected in semistructured interviews.

\section{Setting}

ALFs in a Midwestern state in the US.

\section{Participants}

23 participants who were $38-83$ years of age (mean age $57 \mathrm{y}, 77 \%$ women) and had provided care for relatives with $\mathrm{AD}$ for $0.5-5$ years (mean 2.5 y) before deciding to place them in an ALF in the preceding 6 months.

\section{Methods}

Caregivers participated in semistructured interviews in which they were asked to describe their experiences over time (before the relative's symptoms of memory loss, at the time of diagnosis, during placement, and future care plans). The interviews were audiotaped and transcribed. Data coded in the primary study as "the straw that broke the camel's back" were further categorised according to the 4 elements of a critical incident: a sudden or unexpected happening, the perception of a dangerous or life threatening situation, a sense of loss of control, and disruption of beliefs and values.

\section{Main findings}

All 4 elements of a critical incident were present in the experiences of 19 caregivers. Sudden or unexpected happening: many caregivers expected future placement of their relative in an ALF, but they did not expect it to happen so soon. Some caregivers lived in another state from their relative, and others reported that the symptoms had been hidden from them by their relative. Danger or threat to life: caregivers reported that their relatives were a danger to themselves (eg, eating spoiled food, driving, and wandering). Loss of sense of control: caregivers felt they had lost control of the situation. They tried several alternatives to keep their relatives out of ALFs but were unsuccessful once memory loss progressed. Some caregivers tried moving their relatives into their homes but this disrupted their households. Disruption of beliefs and values: 3 recurrent themes were identified. Caregivers felt responsible for the safety of their relatives with $\mathrm{AD}$ and expressed the feeling that "I couldn't forgive myself if something happened." Care obligations also placed emotional and physical strain on caregivers, and they reported "It was ruining my life." Many caregivers reported burnout from lack of sleep, and their relationships with other family members were negatively affected. For the 4 caregivers for whom all 4 elements of a critical incident were not present, the theme "I had no choice" was identified. They seemed to take a pragmatic rather than emotive view of their situations.

\section{Conclusions}

19 of 23 caregivers reported that the 4 elements of a critical incident were present in the decision to place their relative with Alzheimer's disease in an assisted living facility. Recurrent themes relating to the disruption of beliefs and values were identified: "I couldn't forgive myself if something happened," "it was ruining my life" and "I had no choice."

\section{COMMENTARY}

The study by Liken provides an important understanding of the values underpinning the critical incident leading to institutionalisation of a relative with AD. Previous research has focused on the risk factors leading to institutionalisation, and not the core values underlying these risk factors. Liken's finding of disruption (or conflict) in values and beliefs as a key element leading to placement is consistent with other research that describes caregivers' experiences of conflict in making placement decisions. ${ }^{1}$

Caution must be used in generalising the study results. The study sample was not representative of all caregivers; participants were all Caucasian, primarily daughters and daughters in law $(65 \%)$, and reported higher than average levels of education and income. Thus, further research needs to be done to compare the values precipitating placement decisions between male and female relatives, between children and spouses, among those with differing socioeconomic status, and among those from different cultures.

In this study, relatives were placed in ALFs that provide only supportive care. People who move into nursing homes may have higher levels of physical or cognitive impairment It is not known if the same values underlie placement decisions for both ALFs and nursing homes or if they differ; more research is needed to explore this.

Study results suggest that it is important for clinicians to assess the effect of the caregiving experience on caregivers beliefs and values. Disruption of beliefs and values (eg, experiencing conflicts between protecting the safety of the relative and their right to independence) may be precursors to placement decisions. These are critical times when the provision of emotional, informational, or physical support by clinicians may help with placement decision making and provide necessary relief to the caregiver. Clinicians also have an important role to play in helping relatives work through feelings of conflict, guilt, and loss of control both before and after placement decisions are made.

Jenny Ploeg, RN, PhD McMaster University Hamilton, Ontario, Canada

1 Penrod J, Dellasega C. Caregivers' experiences in making placement decisions. West J Nurs Res 1998;20:706-32. 